\title{
Long time tails in stress correlation functions
}

\author{
M. H. J. Hagen $\quad$ C. P. Lowe \\ D. Frenkel \\ FOM Institute for Atomic and Molecular Physics \\ Kruislaan 407 \\ 1098 SJ Amsterdam \\ The Netherlands
}

\begin{abstract}
The algebraic long time tail of the stress correlation function is observed in a simple lattice Boltzmann model. The amplitude of this tail is compared with the mode coupling expression for the long time tail in the stress correlation function. Agreement is found between mode coupling theory and simulation in both two and three dimensions.
\end{abstract}

\section{Introduction}

In 1970 Alder and Wainwright [1] reported the results of a computer simulation study of the decay of velocity fluctuations in a hard-sphere fluid. These simulations revealed that velocity fluctuations do not decay exponentially, as had been previously assumed, but algebraically. This observation was of great importance because non-exponential decay of the velocity autocorrelation function (velocity ACF) is not compatible with Boltzmann's 'molecular chaos' hypothesis, i.e. the assumption that there is no correlation between the velocity of a particle at time $t$ and the velocity of its collision partners at any later time.

Subsequently, mode coupling [2] and kinetic theories [3] were developed to provide a theoretical framework for the description of long time tails in correlation functions. Both classes of theory reproduce the algebraic decay of 
the velocity $\mathrm{ACF} \phi(t) \sim t^{-d / 2}$, where $d$ is the dimensionality of the fluid, and $t$ the time. In addition, the same theories also predict an algebraic long time tail in the stress correlation function. The mode-coupling theory prediction for the asymptotic form of the stress autocorrelation function is [2]:

$$
\phi_{x y}(t)=\frac{1}{\rho d(d+2)}\left(\frac{d^{2}-2}{(8 \pi \nu t)^{d / 2}}+\frac{1}{(4 \pi \Gamma t)^{d / 2}}\right) \equiv \frac{d_{0}}{t^{d / 2}}
$$

In this equation, $\phi_{x y}(t)$ is the correlation function for the $x y$-component of the stress-tensor, $\rho$ is the number density, $\nu$ is the "bare" kinematic viscosity, and $\Gamma$ is the sound wave damping coefficient. Unlike the long-time tail in the velocity $\mathrm{ACF}$, the algebraic tail in the stress correlation function has thus far not been observed directly neither in simulations nor in experiment, except in a very simple one dimensional model [4] that does not really correspond to a fluid.

In the case of the velocity autocorrelation function, the most accurate numerical results were obtained in simulations of a simplified model for an atomic fluid, namely a lattice-gas cellular automaton of the type introduced by Frisch, Hasslacher and Pomeau [5]. By exploiting some of the special features of the lattice gas, Frenkel and Ernst [6] computed the velocity ACF of a tagged particle with an accuracy that was at least four orders of magnitude better than was hitherto possible. For the velocity ACF of a tagged particle in a lattice gas, it proved possible to perform an average over all possible labelings of the tagged particle. In contrast, no such averaging can be performed in the case of the stress, which is a collective, rather than a single-particle property. As a consequence, the stress correlation function is very noisy. It would seem attractive to try to improve the statistics of the stress ACF by performing some kind of pre-averaging that does reduce the statistical fluctuations but not the way in which stress decays in the latticegas fluid. A natural pre-averaged version of a lattice gas cellular automaton fluid is the so-called lattice-Boltzmann model introduced by McNamara et al. $[7,8]$. The advantage of the lattice Boltzmann model is that one can study the decay of an initial perturbation of the stress without any statistical noise. The disadvantage is that, due to the pre-averaging, it is no longer a truly atomistic model. Moreover, the pre-averaging has killed all spontaneous fluctuations. Hence the way to study the stress ACF is not to watch the decay of spontaneous fluctuations in the stress (there are none), but to make use of Onsager's regression hypothesis and study the decay of an imposed perturbation of the stress. In this paper, we report calculations of the stress $\mathrm{ACF}$, using a lattice-Boltzmann model.

At first sight, it may seem strange to look for long-time tails in a Boltzmann model. After all, in the Boltzmann equation that determines the time evolution of this lattice model, one ignores the correlations between succes- 
sive collisions that, in the kinetic theory description, give rise to long-time tails. Yet, the lattice-Boltzmann model does reproduce the hydrodynamic behavior of a fluid. In the mode-coupling theories of long-time tails in simple fluids, it is precisely the slow decay of hydrodynamic modes that is responsible for the appearance of long-time tails (that are, for this reason, often referred to as hydrodynamic long-time tails).

\section{Lattice-Boltzmann model}

The lattice-Boltzmann model is a pre-averaged version of a lattice-gas cellular automaton (LGCA) model of a fluid. In lattice-gas cellular automaton the state of the fluid at any (discrete) time is specified by the number of particles at every lattice site and their velocity. Particles can only move in a limited number of directions (towards neighboring lattice points) and there can be at most one particle moving on a given 'link'. The time evolution of the LGCA consists of two steps - 1. Propagation: every particle moves in one time step, along its link to the next lattice site. 2. Collision: at every lattice site particles can change their velocities by collision, subject to the condition that these collisions conserve number of particles and momentum (and retain the full symmetry of the lattice). In the lattice-Boltzmann method (see e.g. [9]) the state of the fluid system is no longer characterized by the number of particles that move in direction $\mathbf{c}_{i}$ on lattice site $\mathbf{r}$, but by the probability to find such a particle. The single-particle distribution function $n_{i}(r, t)$, describes the average number of particles at a particular node of the lattice $\mathbf{r}$, at a time $t$, with the discrete velocity $\mathbf{c}_{i}$. The hydrodynamic fields, mass density $\rho$, momentum density $\mathbf{j}$, and the momentum flux density $\Pi$ are simply moments of this velocity distribution:

$$
\rho=\sum_{i} n_{i}, \quad \mathrm{j}=\sum_{i} n_{i} \mathbf{c}_{i}, \quad \Pi=\sum_{i} n_{i} \mathbf{c}_{i} \mathbf{c}_{i} .
$$

The lattice model used in this work is the four dimensional Face-Centered Hyper Cubic (FCHC) lattice. A two or three dimensional model can then be obtained by projection in the required number of dimensions. This FCHC model is used because three-dimensional cubic lattices do not have a high enough symmetry to ensure that the hydrodynamic transport coefficients are isotropic.

The time evolution of the distribution functions $n_{i}$ is described by the discretized analogue of the Boltzmann equation [10]:

$$
n_{i}\left(\mathbf{r}+\mathbf{c}_{i}, t+1\right)=n_{i}(\mathbf{r}, t)+\Delta_{i}(\mathbf{r}, t),
$$


where $\Delta_{i}$ is the change in $n_{i}$ due to instantaneous molecular collisions at the lattice nodes. The post-collision distribution $n_{i}+\Delta_{i}$ is propagated in the direction of the velocity vector $c_{i}$. A complete description of the collision process is given in [11]. The main effect of the collision operator $\Delta_{i}(\mathbf{r}, t)$ is to relax the non-equilibrium part of the momentum flux. The full, non-linear expression for equilibrium part of the local momentum flux density $\Pi^{\mathbf{e q}}$ is given by:

$$
\Pi^{\mathrm{eq}}=p \mathbf{I}+\rho \mathbf{u u},
$$

with $p$ the local pressure, I the unit tensor, and $\mathbf{u}$ the local fluid velocity. In the linearized version the equilibrium part of the momentum flux density is given by:

$$
\Pi^{\mathrm{eq}}=p \mathbf{I} \text {. }
$$

The rate of stress relaxation, or equivalently, the kinematic viscosity $\nu$, can be chosen freely. In the linear lattice-Boltzmann model (eq. 5) $\Pi_{x y}$ can only decay exponentially. To observe the long time behavior of the stress ACF, a coupling to the momentum is essential. The second term in eq. (4) which is usually only taken into account to study high Reynolds number flow, does exactly this.' In order to observe the long time tail in the stress ACF, the full non-linear stress tensor had to be used in the simulation.

As the lattice-Boltzmann model is purely dissipative, microscopic fluctuations in the fluid are not included. Such fluctuations can be incorporated in the lattice-Boltzmann model by adding a suitable random noise term to the stress [12]. However, for the present work, such fluctuations are not essential for the phenomenon under study yet would seriously deteriorate the statistical accuracy of our calculations.

The stress in the system, which is a collective property is given by:

$$
\boldsymbol{\Sigma}=\sum_{\mathbf{r}}(\Pi-p \mathbf{I})
$$

For the sake of convenience, we consider only one component of the traceless symmetric part of the stress tensor viz. the $x y$ component. Other components give rise to the same correlation functions. We compute the stress ACF by correlating the initial perturbation of the stress with the stress at some later time $t$ :

$$
\phi_{x y}(t)=\frac{\left\langle\Delta \Sigma_{x y}(0) \Delta \Sigma_{x y}(t)\right\rangle}{\left\langle\left(\Delta \Sigma_{x y}(0)\right)^{2}\right\rangle},
$$

where $\Delta \Sigma_{x y}=\Sigma_{x y}(t)-\Sigma_{x y}(\infty)$. It is important to subtract the steady state $(t=\infty)$ value of the stress tensor because, in a finite system, the initial stress perturbation will relax to a uniform velocity field with an associated stress given by eq. (4):

$$
\Sigma_{x y}(\infty)=\frac{\Sigma_{x y}(0)}{\rho V}
$$


where $V$ is the volume of the fluid. In a LGCA, where the stress is purely kinetic in origin, the stress at site $\mathbf{r}$ at time $t$ is uncorrelated to the stress at that same time at any other lattice point. In our calculations we have therefore chosen to consider the simplest possible initial condition viz. a small perturbation of the stress at one lattice site only.

\section{Results}

Having set up the system with an initial local stress perturbation, we followed the time-evolution of the total stress of the system using the dynamics of the lattice-Boltzmann model. In fact, we did the simulations both for the linearized and the, non-linear expression for the stress tensor.

In order to be able to compare the tail amplitude as obtained from the simulations with the theoretical expression (Eq. (1)) we need to know the sound damping coefficient $\Gamma$. At the Boltzmann level, this quantity is given by: $\Gamma=2(d-1) \nu / d+\zeta$ with $\zeta$ the kinematic bulk viscosity. $\nu$ and $\zeta$ were 'measured' by setting up a sound wave in the system and measuring the decay of that wave in the long wavelength limit [14]. $\Gamma, \zeta$ and $\nu$ were computed for a range of imposed kinematic viscosities between 0.01 and 0.50 .

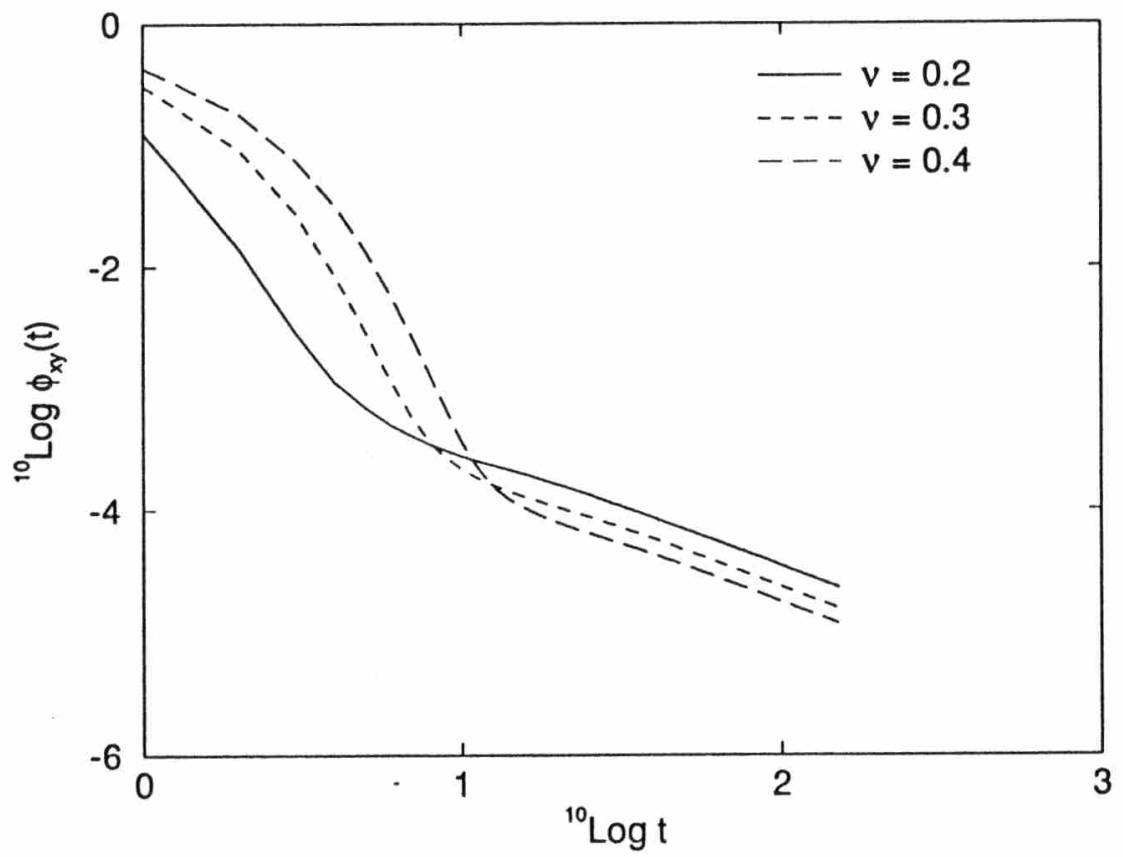

Figure 1: The stress autocorrelation function of a two dimensional lattice-gas fluid. 
The simulations in two dimensions were performed on a system of $250 \times$ 250 lattice sites. For this size of simulation box we followed the stress ACF for 140 time steps. This upper limit was chosen because, after this time, interference occurred due to sound waves that cross the periodic system. In figure 1, we show the stress ACF of the lattice Boltzmann model for several different values of the kinematic viscosity and the non-linear expression for the stress. For this model, we do indeed observe a clear algebraic decay of the stress ACF. As expected for a two-dimensional fluid, the exponent of the algebraic long-time tail was -1 . In contrast, no algebraic tail is observed if the non-linear terms in the stress are ignored. This is understandable because in the linearized model there is no mechanism by which the different modes can couple.

The limiting value $d_{0}$ was determined by plotting $t \phi_{x y}(t)$ as a function of $1 / t$. The intercept for $1 / t=0$ yields the desired amplitude. The results of this analysis are shown in figure 2. In this figure, we also show the theoretical tail coefficient given by Eqn. (1). Figure 2 shows that the mode-coupling predictions of the tail coefficient are in almost quantitative agreement with

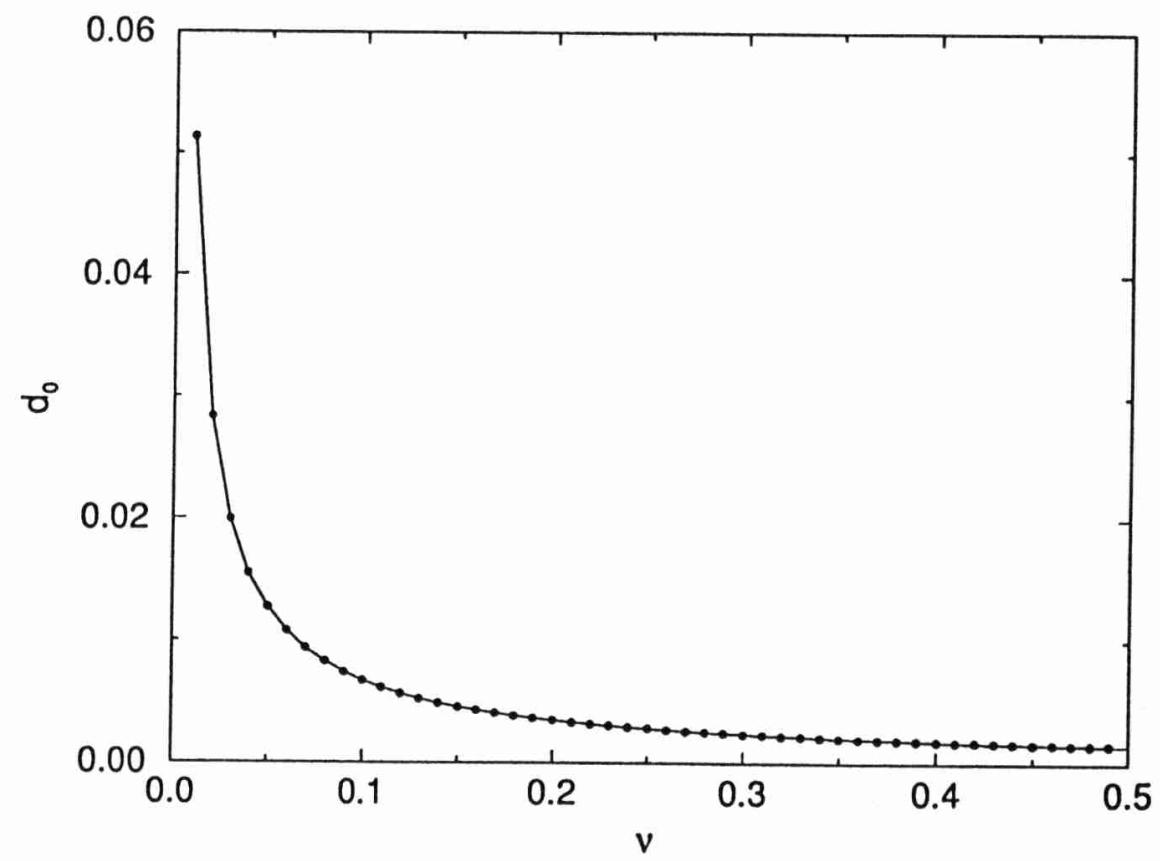

Figure 2: The tail coefficient for a two dimensional lattice gas fluid, as a function of the kinematic viscosity $\nu$. The points are the results of simulations of the latticeBoltzmann model, while the drawn curve corresponds to the prediction of mode coupling theory. 
the simulation results. In fact, there is a very small discrepancy between the mode-coupling theory predictions and the simulation results. However, this discrepancy is consistent with inaccuracy in the determination of $d_{0}$.

The simulations in three dimensions were done for a system $90 \times 90 \times 90$ lattice sites. For this size simulation box we followed the stress ACF for times up to $t=50$. After this time interference due to the round-trip of sound waves occurred. In the three-dimensional fluid, we also find algebraic decay of the stress ACF in the non-linear lattice-Boltzmann model only. The algebraic tail is characterized by an exponent -1.5 , as expected. Figure 3 shows the stress ACF of the $3 D$ lattice Boltzmann fluid for several values of the kinematic viscosity.

We performed almost the same extrapolation procedure as described above to determine the amplitude of the long-time tail. Specifically, we plotted $t^{3 / 2} \phi_{x y}(t)$ as a function of $1 / t$. As before, the amplitude of the algebraic tail $d_{0}$ is obtained from the intercept of $t^{3 / 2} \phi_{x y}(t)$ in the limit $1 / t \rightarrow 0$.

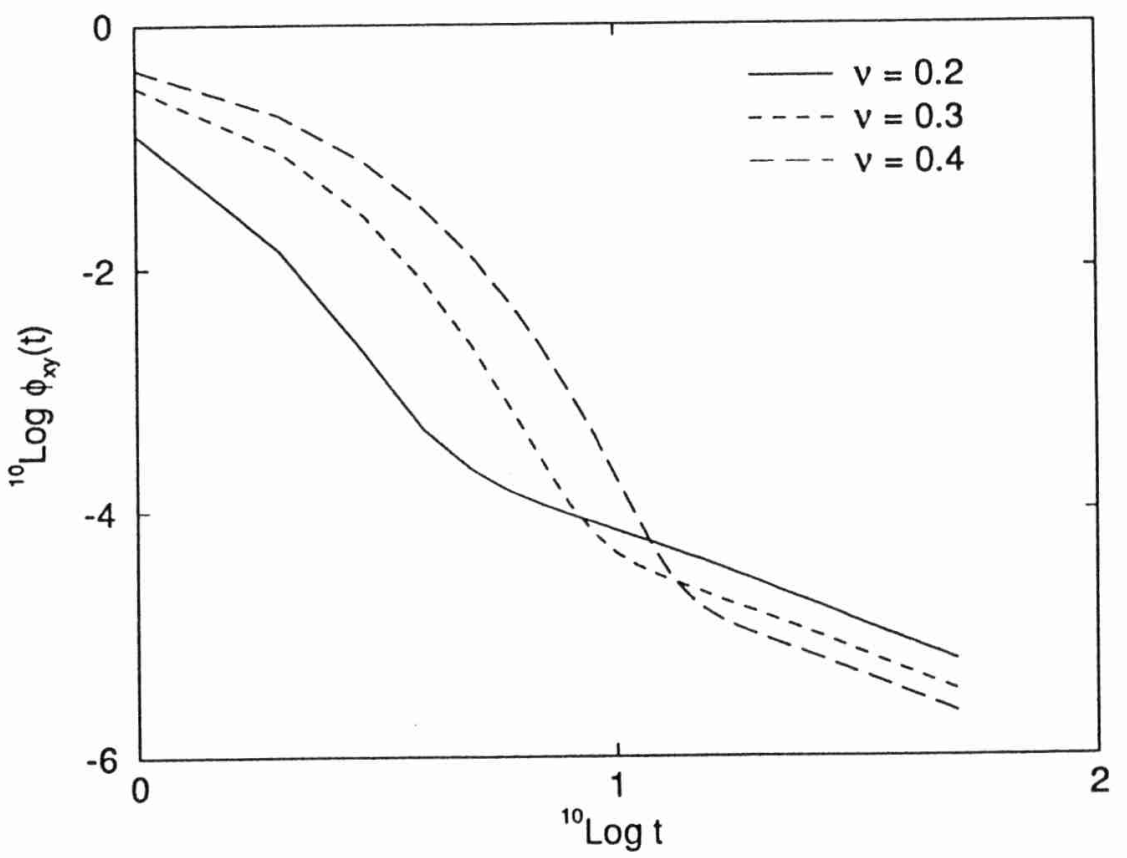

Figure 3: The stress autocorrelation function of a threedimensional lattice-gas fluid. 
Figure 4 shows a comparison of the tail coefficient obtained from the simulations, with the corresponding mode-coupling prediction (Eqn. (1)). As can be seen from this figure, there is again almost quantitative agreement between mode-coupling theory and the simulation results. The small discrepancy is caused by the short simulation time and is expected to disappear if longer simulations in a larger system could be performed.

In the three dimensional system we have also computed to what extent the long-time tail in the stress ACF changes the "bare" kinematic viscosity $\nu$ which was computed at the Boltzmann level. This is done by using the Green-Kubo formula for the viscosity [15]:

$$
\nu_{\mathrm{lydro}}(t) \sim \frac{1}{2} \phi_{x y}(0)+\sum_{t^{\prime}=1}^{t} \phi_{x y}\left(t^{\prime}\right)
$$

Asymptotically, $\iota_{\text {hydro }}(t) \sim t^{-1 / 2}$ (from Eq. (1)), and in this way extrapolation was performed to find $\nu_{\text {liydro }}=\lim _{t \rightarrow \infty} \nu_{\text {liydro }}(t)$.

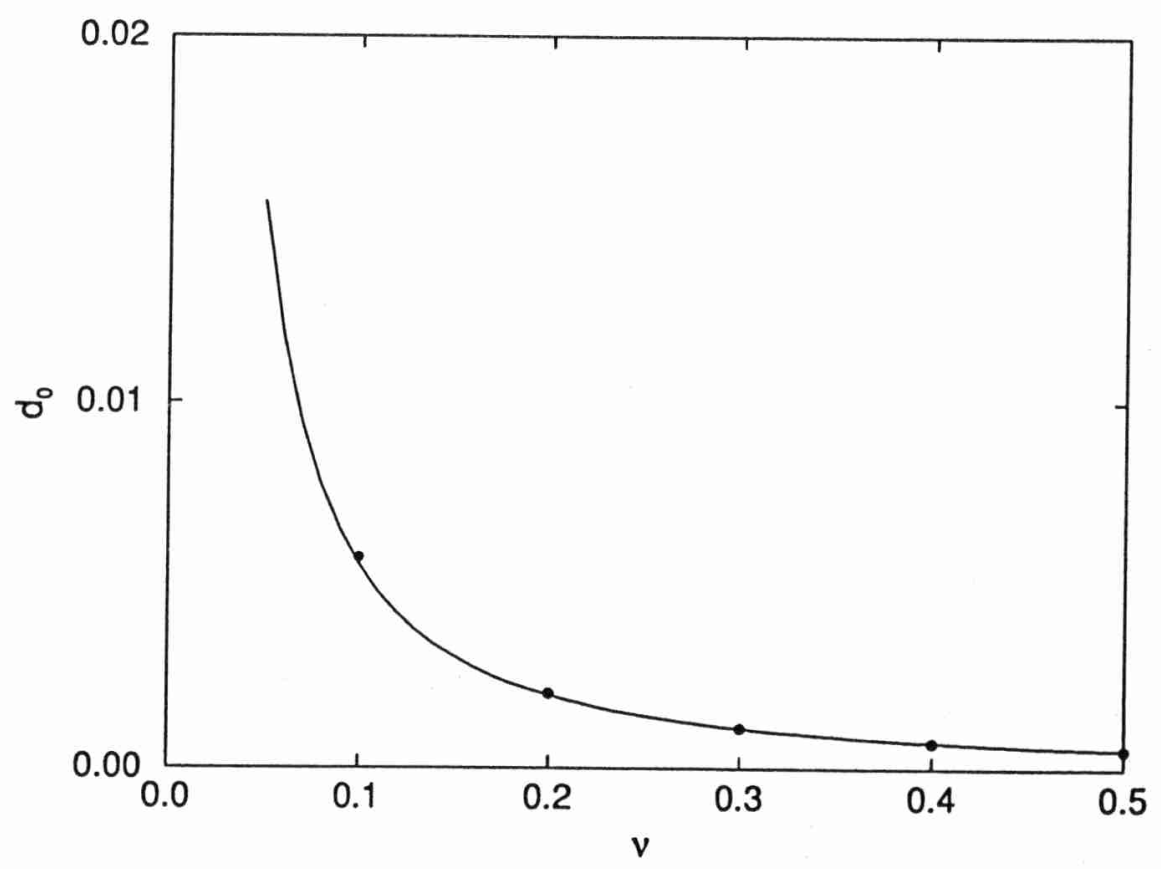

Figure 4: The tail coefficient for a three dimensional lattice-gas fluid, as a function of the kinematic viscosity $\nu$. The points are the results of simulations of the lattice-Boltzmann model, while the drawn curve line corresponds to the prediction of mode coupling theory. 
The result of this calculation is shown in table 1. Note that the algebraic

\begin{tabular}{|c|c|}
\hline$\nu$ & $\left(\nu_{\text {liydro }}-\nu\right) / \nu$ \\
\hline 0.1 & 0.171 \\
0.2 & 0.079 \\
0.3 & 0.053 \\
0.4 & 0.041 \\
0.5 & 0.033 \\
\hline
\end{tabular}

Table 1: The relative effect of the hydrodynamic long time tail on the viscosity in three dimensions.

long-time tail results in a small re-normalization of the viscosity. The same calculation was not performed in two dimensions, because the re-normalized kinematic viscosity diverges in that case.

\section{Conclusions}

We have computed the stress ACF of a lattice Boltzmann fluid and compared the results with mode coupling theory. We find that both the exponent of the algebraic long time tail $(-d / 2)$ and the its amplitude $\left(d_{0}\right)$ are in essentially quantitative agreement with mode coupling theory. The computation of the time dependent viscosity in three dimensions shows that, at least for the simple lattice-gas model studied in this work, the hydrodynamic longtime tail of the stress ACF results in a small correction to the Boltzmann prediction of the kinematic viscosity.

\section{Acknowledgements}

We gratefully thank M.H. Ernst and H. van Beijeren for stimulating correspondence. The work of the FOM Institute is part of the scientific program of FOM and is supported by the Nederlandse Organisatie voor Wetenschappelijk Onderzoek (NWO). Computer time on the CRAY-C98/4256 at SARA was made available by the Stichting Nationale Computer Faciliteiten (Foundation for National Computing Facilties). 


\section{References}

[1] B. J. Alder and T. E. Wainwright, Phys. Rev. A. 1, 18 (1970).

[2] M. H. Ernst, E. H. Hauge and J. M. J. van Leeuwen, Phys. Rev. Lett. 25, 1254 (1970).

[3] J. R. Dorfman and E. G. D. Cohen, Phys. Rev. Lett. 25, 1257 (1970).

[4] T. Naitoh and M. H. Ernst, Molecular Simulation. 12, 197 (1994).

[5] U. Frisch, B. Hasslacher and Y. Pomeau, Phys. Rev. Lett. 561505 (1986).

[6] D. Frenkel and M. H. Ernst, Phys. Rev. Lett. 632165 (1989).

[7] G. R. McNamara and G. Zanetti, Phys. Rev. Lett. 61, 2332 (1988).

[8] F. Higuera, S. Succi, and R. Benzi, Europhys. Lett. 9, 345 (1989).

[9] G. R. McNamara and B. J. Alder, in Microscopic Simulation of Complex Hydrodynamic Phenomena, edited by M. Mareschal and B. L. Holian (Plenum, New York, 1992).

[10] U. Frisch, D. d'Humières, B. Hasslacher, P. Lallemand, Y. Pomeau, and J. -P. Rivet, Complex Systems 1, 649 (1987).

[11] A. J. C. Ladd, J. Fluid Mech. 271, 285 (1994).

[12] A. J. C. Ladd, Phys. Rev. Lett. 70, 1339 (1993).

[13] M. A. van der Hoef and D. Frenkel, Phys. Rev. Lett. 661591 (1991).

[14] D. d'Humières and P. Lallemand, Complex Systems 1, 599 (1987).

[15] J. W. Dufty and M. H. Ernst, J. Phys. Chem. 93, 7015 (1989).

[16] M. H. Ernst and H. van Beijeren (private communication). 